\title{
Novel Texture Controlling of Mg Alloys
}

\author{
Bin Jiang, Guangsheng Huang, Jiangfeng Song, Dingfei Zhang, \\ and Fusheng Pan
}

\section{Extended Abstract}

Magnesium (Mg) alloys have attracted considerable attention for a promising application in the automotive and electronics owing to their high specific strength and high electromagnetic shielding. However, the application of wrought $\mathrm{Mg}$ alloys has been limited by poor room temperature ductility. It was ascribed to the large difference in critical resolved shear stresses (CRSS) between basal and prismatic slip in hexagonal close-packed (hcp) crystal structure in $\mathrm{Mg}$ alloy. This results in a lack of the active slip systems and can hardly offer an arbitrary shape change at the grain level.

Conventionally extruded $\mathrm{Mg}$ alloy sheets possess poor mechanical properties due to the strong basal texture where $c$-axes of the grains are predominantly aligned parallel to the sheet normal. This brings about a poor deformation capability of sheet thinning and a stronger anisotropy and consequently results in a limited number of available plastic deformation modes. In this work, a novel extrusion approach to get high strength magnesium alloy plates will be introduced through differential speed processing. A suitable constitutive model of differential speed extrusion is established to ameliorate the texture-dependent mechanical properties. The velocity evolutions of the extruded sheets at near-surface and mid-layer regions are different due to the extra asymmetric shear deformation. This simple shear enforces the near-surface microstructure to exhibit more dynamically recrystallized grains having the c-axis tilted toward the extrusion direction. The yield stress of AZ31 alloy sheet has been increased from 161.2 to $179.9 \mathrm{MPa}$, and the elongation has been improved from 15.4 to $20.1 \%$. Moreover, as for the high strength AZ61 alloy sheets, the ultimate tensile strength was increased from 387.9 to 427.1 MPa and the yield stress was improved from 147.7 to 195.9 MPa. Grain refinement and tilted weak basal texture obtained by differential speed extrusion process. This approach is an efficient substitute to increase the textureinduced softening and ductility and thus favorable for the thin sheet fabrication. 